\title{
A small molecular agent YL529 inhibits VEGF-D-induced lymphangiogenesis and metastasis in preclinical tumor models in addition to its known antitumor activities
}

Youzhi $\mathrm{Xu}^{1,2+}$, Wenjie Lu ${ }^{1,2+}$, Peng Yang ${ }^{3+}$, Wen Peng ${ }^{2,4+}$, Chunting Wang ${ }^{2}$, Manli $\mathrm{Li}^{2}$, Yan $\mathrm{Li}^{2}$, Guobo $\mathrm{Li}^{2}$, Nana Meng ${ }^{2}$, Hongjun Lin ${ }^{2}$, Lixin Kan², Siying Wang ${ }^{2}$, Shengyong Yang ${ }^{2}$, Luoting $\mathrm{Yu}^{2^{*}}$ and YingLan Zhao ${ }^{2^{*}}$

\begin{abstract}
Background: The lymph node metastasis is a key early step of the tumor metastatic process. VEGFD-mediated tumor lymphangiogenesis plays a key role, since down-regulation of p-VEGFR-3 could block the lymph node metastasis. YL529 has been reported to possess potent anti-angiogenesis and antitumor activities; however, its roles in tumor-associated lymphangiogenesis and lymphatic metastasis remain unclear.

Method: We investigated the effect of YL529 on tumor-associated lymphangiogenesis and lymph node metastasis using in vitro lymph node metastasis models and in vivo subcutaneous tumor models in C57 BL/6 mice.

Result: We found that YL529 inhibited VEGF-D-induced survival, proliferation and tube-formation of Human Lymphatic Endothelial Cells. Furthermore, in established in vitro and in vivo lymph node metastasis models using VEGF-D-LL/2 cells, YL529 significantly inhibited the tumor-associated lymphangiogenesis and metastasis. At molecular level, YL529 down-regulated p-VEGFR-3, p-JNK and Bax while up-regulated Bcl-2.

Conclusion: YL529 provided the therapeutic benefits by both direct effects on tumor cells and inhibiting lymphangiogenesis and metastasis via the VEGFR-3 signaling pathway, which may have significant direct clinical implications.
\end{abstract}

Keywords: YL529, Lymphangiogenesis, Metastasis, VEGF-D, VEGFR-3

\section{Background}

Tumor metastasis is the key and final cause of cancer mortality [1-3]. It usually occurs via the hematogenic and the lymphogenic metastasis routes $[4,5]$, and the latter is considered to be a very important route contributing to metastasis of solid tumors [6].

Tumor lymphangiogenesis is regulated by many important factors, such as vascular endothelial growth factor (VEGF) and its receptor subtype members (VEGFRs). VEGFR-1 3 are almost exclusively located on the surface

\footnotetext{
* Correspondence: zhaoyinglan@scu.edu.cn; ylting_cd@163.com

${ }^{\dagger}$ Equal contributors

${ }^{2}$ State Key Laboratory of Biotherapy and Cancer Center, West China Hospital, West China Medical School, and Collaborative Innovation Center for Biotherapy, Sichuan University, 17\#, 3rd Section, Ren min South Road, Chengdu 610041, China

Full list of author information is available at the end of the article
}

of vascular endothelial cells and lymphatic vessels in normal tissues and are up-regulated only during embryonic and tumor. Among all of VEGFs and VEGFRs, the Vascular endothelial growth factor D (VEGF-D) is indispensable for development of the lymphatic system and VEGFR-3 has also been implicated as the major effectors of lymphangiogenesis and regulates lymphatic vessel growth $[1,7]$; VEGF-C and VEGF-D are the ligands for the tyrosine kinases VEGFR-3 and VEGFR 2, which mainly regulates the growth of lymphatic vessels via their receptor VEGFR-3, and partly regulates the growth of blood vessels via VEGFR-2 $[8,9]$. Since literatures have showed that tumor-induced lymphangiogenesis driven by VEGF-C and VEGF-D-induced activation of VEGFR-3 could promote regional lymph node metastasis in multiple solid tumors 
[10-13]. Consistently, tumor cells with up-regulated VEGF-C and VEGF-D could increase the intratumoral and peritumoral lymphangiogenesis and exacerbate metastasis to local lymph nodes and distant organs [14]. And some researchers have reported that VEGF-C plays an important role in the process of lung cancer metastasis, but there are just a few reports about VEGF-D as the ligand for VEGFR-3 in the tumor metastasis process in vivo $[1,2]$. Therefore, targeting of VEGF-C or VEGF-D/VEGFR-3 could potentially block the lymphatic metastasis.

Current studies suggest that therapeutic strategies targeting tumor lymphangiogenesis via the VEGF/VEGFR kinase axis are promising approaches for the treatment of cancer lymphogenic metastasis. However, quite a few target therapies show toxicity and have only moderate response rates for tumor treatment.

A number of small molecules, which could inhibit the intrinsic tyrosine kinase activity of VEGFR, have been reported previously with a range of nanomolar potencies, specificities, and pharmacokinetic properties [15-17]. Our group also focused on developing a small molecular compound that potently and selectively blocks the VEGF-D/ VEGFR3 receptor system after oral administration, suitable for the chronic therapy of VEGF-D/VEGFR3dependent pathological lymphangiogenesis. We previously described YL529, a small molecular anti-cancer agent synthesized by our laboratory, inhibits tumor neovascularization and cell proliferation in a panel of cell lines and in tumor-bearing mouse models. YL529 has demonstrable potent antitumor and anti-angiogenic properties against human umbilical vein endothelial

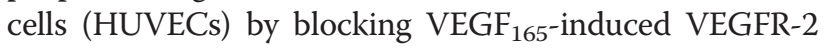

autophosphorylation [18]. YL529 also inhibited the phosphorylation of VEGFR-3. However, it is still unclear whether YL529 could effectively inhibit lymphangiogenesis and the associated lung and lymphatic metastasis, or whether YL529 could potentially improve the survival of the affected individuals.

In present study,we choose to detect the expression of VEGF-D in VEGF-D-LL/2 cell after YL529 treatment because other researchers in our group have been successfully established the over-expression VEGF-D in Lewis lung cancer cells VEGF-D-LL/2 cell in vitro. And it is true that tumor metastatic models have been successfully established via injecting over-expression VEGF-D Lewis lung cancer cells (VEGF-D-LL/2) in C57 BL/6 mice [19]. Our current study aimed to further address these issues, and we found that YL529 blocked VEGFD-induced lymphangiogenesis and inhibited tumor growth efficiently at the dosage of $150 \mathrm{mg} / \mathrm{kg} /$ day or even less, as a result, affected mice have significantly better survival rate. This novel finding may have significant direct clinical implications.

\section{Methods}

\section{Preparation of YL529}

The route of synthesis of YL529 (N-methyl-4-(4-(3(trifluoromethyl)benzamido) phenoxy)picolinamide4methylbenzenesulfonate) was provided by State Key Laboratory of Biotherapy, Sichuan University (Sichuan, China), its structural formula is shown in Fig. 1a, YL529 were analyzed and identified by high performance liquid chromatography (HPLC, Waters, MA, USA) and nuclear magnetic resonance (NMR). For all in vitro assays, YL529 was prepared as stock solution in dimethyl-sulphoxide

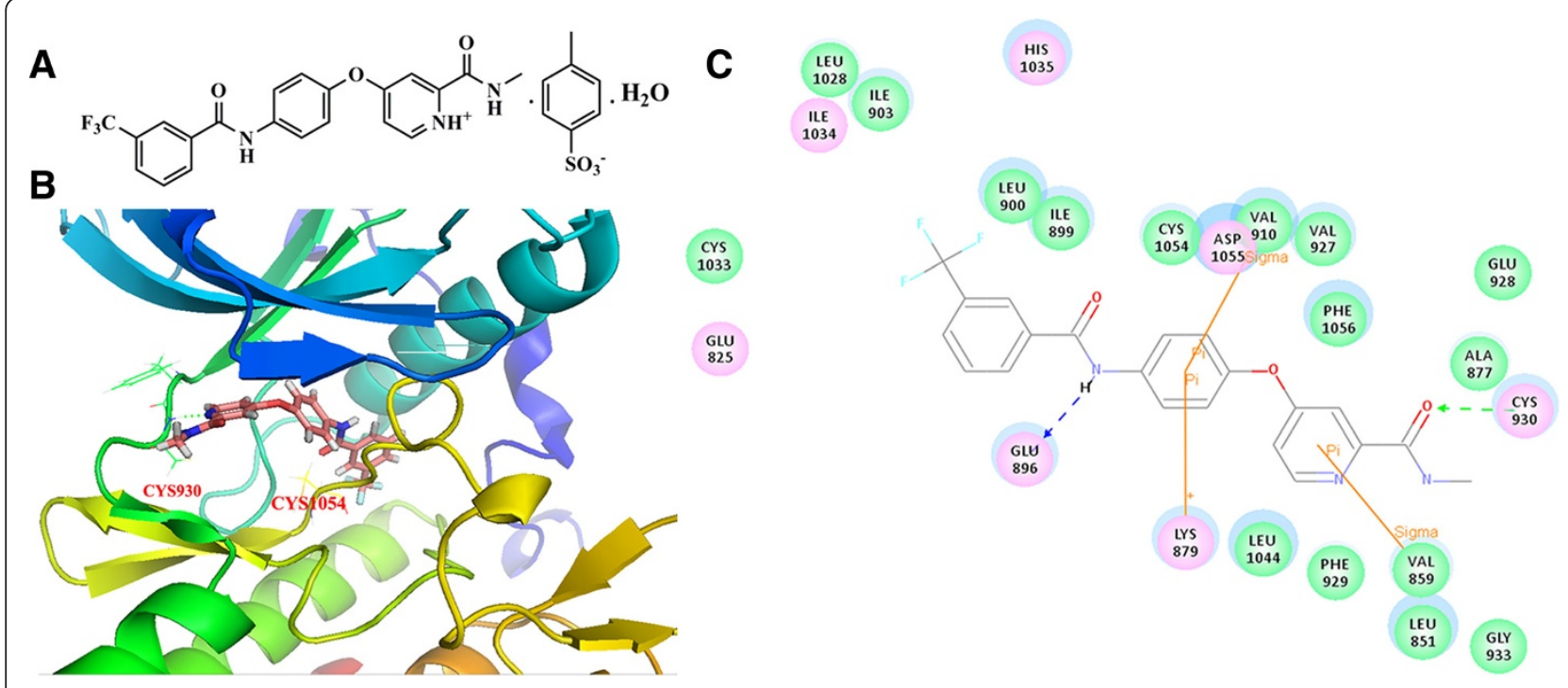

Fig. 1 The chemical structure of YL529 and its interaction with VEGFR3. a The chemical structure of YL529; b and c The interactions between YL529 and VEGFR3 were shown in 3-D structure and 2-D map 
(DMSO) at final DMSO concentration $0.05 \%(\mathrm{~V} / \mathrm{V})$ and diluted in the cell culture medium. For in vivo studies, YL529 was dissolved in ultrapure water with $0.5 \%$ sodium carboxymethylcellulose (CMC-Na) combined with $2 \%$ Tween-20, then administered by oral gavages at $10 \mathrm{ml} / \mathrm{kg} /$ day.

\section{Homology modeling and molecular docking methods}

The sequence of human VEGFR3 (Genbank accession number: AAO89505.1) was extracted from the NCBI protein sequence database. The crystal structure of VEGFR2 (PDB code: 4ASD) was used as a template for human VEGFR3 homology modeling; the sequence identity between VEGFR2 and VEGFR3 is larger than $70 \%$. The homology model of VEGFR3 was generated using the MODELER program implemented in Discovery Studio (DS) 3.1 software package.

The established VEGFR3 homology model was then used for the following docking study. A sphere containing the key residues in VEGFR3 (including CYS930, ALA877, VAL859, PHE929, LEU1044, LYS879, ASP1055, CYS1054, VAL910, GLU896, ILE899, and LEU900) was defined as the binding site. GOLD 5.0 was used for molecular docking since it was an excellent docking program. Gold Score was selected as the score function; number of dockings was set as 30; and the other parameters were set as default. The docking results were shown in Fig. $1 \mathrm{~b}$ and c.

\section{Antibody and reagents}

The primary antibodies VEGF-D, LYVE-1, $\beta$-actin were purchased from Santa Cruz Biotechnology (Santa Cruz, CA); VEGFR-3/phospho-VEGFR-3, JNK phospho-JNK, $\mathrm{Bcl}-2$ and Bax antibodies were purchased from Cell Signaling Laboratories (Beverly, MA). The terminal deoxynucleotidyl transferase mediated nick-end labeling (TUNEL) assay kit was purchased from Promaga Company (Madison, WI). DMSO, Tween-20, Gelatin and CMC-Na were purchased from Sigma Chemical Co, (St. Louis, MO); Matrigel was purchased from BD Pharmingen (La Jolla, CA).

\section{Cell culture}

Human lymphatic endothelial cells (HLECs) were purchased from the ScienCell ${ }^{\mathrm{Tm}}$ Research Laboratories and cultured in endothelial cell medium (ECM) containing $5 \%$ fetal bovine serum (FBS), $1 \%$ endothelial cell growth supplement (ECGS), $100 \mathrm{IU} / \mathrm{mL}$ penicillin and $100 \mu \mathrm{g} / \mathrm{mL}$ streptomycin. Lewis lung carcinoma LL/2 cells were obtained from American Type Culture Collection (ATCC) and cultured in DMEM containing $10 \%$ FBS, $100 \mathrm{IU} / \mathrm{mL}$ penicillin and $100 \mu \mathrm{g} / \mathrm{mL}$ streptomycin. Subcultures were performed with trypsin-EDTA. All cells were incubated in an atmosphere of $5 \% \mathrm{CO}_{2}$ at $37^{\circ} \mathrm{C}$.
The establishment of VEGF-D high-expressing Lewis lung cell lines

The transfected murine LL/2 Lewis lung carcinoma cells with the pcDNA3.1 $(+)$ expression vector containing mouse VEGF-D (VEGF-D-LL/2 cells) or pcDNA3.1 (vector alone) were transfected as reported before obtained in our laboratory [20-22]. The cell lines were maintained in an atmosphere of $5 \% \mathrm{CO}_{2}$ at $37{ }^{\circ} \mathrm{C}$ in cell culture medium DMEM supplemented with $10 \%$ fetal bovine serum. Western blot analysis was performed to analyze the expression of recombinant VEGF-D of these cells.

\section{Cell viability assay}

Cell viability assays were performed using CCK-8 kit [23]. Briefly, VEGF-D-LL/2 cells, parental LL/2 cells and HLECs were treated with various concentrations of YL529 in 96-well culture plates for $48 \mathrm{~h}$. CCK- 8 was added to the cells and the plate was incubated for an additional 2-4 h. The optical density (OD) was then measured at $450 \mathrm{~nm}$ using a Spectra MAX M5 microplate spectrophotometer (Molecular Devices, Sunnyvale, CA).

\section{Scratch-induced migration assay}

The anti-metastasis effect of YL529 was determined using a scratch-induced cell migration assay in vitro [24, 25]. Briefly, $1 \times 10^{5}$ HLECs were plated in 6 well plates and synchronized by serum-free ECM medium for $8 \mathrm{~h}$, then used a micropipette tip to create a $2 \mathrm{~mm}$-wide linear gap. Cells were washed to remove non-adherent cells and further incubated with fresh ECM medium containing or lacking YL529. An inverted microscope (Carl Zeiss, Germany) was used to photograph after YL529 treatment for another $48 \mathrm{~h}$.

\section{Transwell migration assay}

Transwell migration assay was adopted to indicate the anti-metastasis effect of YL529 [26]. Briefly, the transwell were pre-coated with $25 \%$ Matrigel Matrix containing growth factors (BD Biosciences, Bedford, MA) for $30 \mathrm{~min}$ at $37{ }^{\circ} \mathrm{C}$. And then the bottom chambers were filled with $600 \mu \mathrm{l} \mathrm{ECM}$ medium and the top chambers were seeded with $4 \times 10^{4}$ cells HLECs $(100 \mu \mathrm{l} /$ well $)$. The top and bottom chambers were incubated for another $24 \mathrm{~h}$. Then cells on the top surface of the membrane were scraped with a cotton swab. Cells on the bottom side of the membrane were fixed with $4 \%$ paraformaldehyde and stained with $0.1 \%$ crystal violet (Sigma-Aldrich, USA). An inverted microscope (Zeiss, Axiovert 200, Germany) was used to obtain the images and the invading cells were quantified.

\section{Tube formation assay}

The antilymphogenic effects of YL529 were analyzed in vitro using a tube-formation assay in HLECs. Briefly, 96-well plate was pre-incubated with $100 \mu \mathrm{l}$ per well of 
Matrigel Matrix at $37^{\circ} \mathrm{C}$ for $30 \mathrm{~min}$, HLECs were seeded at a density of $3 \times 10^{4}$ cells per well in ECM medium. And after cultured in the presence or absence of designed concentrations of YL529 on Matrigel Matrix at $37{ }^{\circ} \mathrm{C}$ another $6 \mathrm{~h}$, tube formation by endothelial cells was evaluated and photographed under an inverse microscope (Zeiss, Axiovert 200, Germany).

\section{Western blot analysis}

Standard western blot analysis was performed [23]. Briefly, Cell lysates were washed with phosphate buffered saline (PBS) and lysed in RIPA (radioimmunoprecipitation assay) buffer. Then lysates were centrifuged at $12000 \mathrm{~g}$ for $30 \mathrm{~min}$ at $4{ }^{\circ} \mathrm{C}$. The Bio-Rad Protein Assay kit (Bio-Rad Laboratories) was used to determine the samples protein concentration according to the manufacturers' recommendations. The lysates were dissolved in $5 \times$ SDS sample buffer and denatured, then subjected to $6 \%$ to $12 \%$ SDS-PAGE (sodium dodecyl sulfate polyacrylamide gel electrophoresis) according to molecular weight and transferred onto PVDF (polyvinylidene fluoride) (Bio-Rad, Hercules, CA) membranes. Membranes were blocked for $1 \mathrm{~h}$ in $5 \%$ dried milk in TBS/T at room temperature and incubated overnight at $4{ }^{\circ} \mathrm{C}$ with the primary antibodies and horseradish peroxidase-conjugated secondary antibodies. Protein bands were visualized with enhanced chemiluminescent substrate (Amersham Biosciences Corp., Piscataway, NJ).

Pharmacokinetic characteristics analyses of YL529 in mice C57 BL/6 mice ( $n=3$ per time point) were administered $150 \mathrm{mg} / \mathrm{kg}$ YL529 orally. Blood samples of the mice were collected at appropriate intervals and the plasma concentration of YL529 was analyzed by HPLC (Waters, MA, USA). The pharmacokinetic characteristics and parameters were analyzed using Pharmacokinetic Software of Drug and Statistics (DAS, edited and published by the Mathematical Pharmacology Professional Committee of China, Shanghai, China).

\section{Effect of YL529 on lymph metastasis in mice syngeneic models}

Seven-week-old female C57 BL/6 mice (Beijing animal center, Beijing, China) were used. VEGE-D-LL/2 cells $\left(1 \times 10^{6}\right)$ were injected intramuscularly and subcutaneously in hind limb of mice. The mice were randomized into 5 groups ( $n=10$ per groups) and orally administered 37.5, 75 and $150 \mathrm{mg} / \mathrm{kg} /$ day YL529, vehicle and saline alone (N.S.), respectively for 14 days when tumors became visible the $10^{\text {th }}$ day after cells were implanted. Tumor growth and mice weight was monitored every 3 days. When animals were sacrificed with $\mathrm{CO}_{2}$ gas at the end of drug administration, tumor tissues, lung organs and local lymph nodes were removed, weighted and calculated by manual. Tumor size was determined by measuring the largest and perpendicular diameters every 3 days, tumor volume was calculated using the formula: volume $\left(\mathrm{mm}^{3}\right)=0.5 \times$ length $\times$ width $^{2}$. In addition, the same subcutaneously tumor models were used to monitor the survival time of experimental mice after drug administration. All animals experiments performed were in accordance with the guidelines of the institute's Animal Care and Use Committee of Sichuan University (Chengdu, China).

\section{Histological analysis for tumor tissue}

Tumor tissues from vehicle and $150 \mathrm{mg} / \mathrm{kg}$ YL529 groups were fixed in $4 \%$ paraformaldehyde, dehydrated and embedded in paraffin [27]. Sections of these tissues were subsequently incubated with LYVE-1 and corresponding second antibodies and visualized using peroxidase-DAB. TUNEL staining was also performed for fixed tissues (Promega, USA). Quantification was done as described [28]. Briefly, the microvessel counting was done in representative $200 \times$ fields or three high-power $(400 \times)$ fields of the highest vascular density.

\section{Safety profile of YL529 in vivo}

To evaluate the safety profile of YL529 $(150 \mathrm{mg} / \mathrm{kg})$ in vivo, we have observed the gross measures such as weight loss, life span, behavior and feedings. Moreover, we have also investigated the mortality and clinical signs of C57BL/6 mice throughout oral administration period. In addition, blood samples and heart, liver, spleen, lung, and kidney tissues of mice were collected; the histopathological, serum biochemistry and hematological analysis were done to observe the possible pathological changes. In addition, we also evaluated the safety profiles of YL529 after oral administration with high dose $(6000 \mathrm{mg} / \mathrm{kg})$ for 14 days.

\section{Statistical analysis}

Data was expressed as mean \pm SD/SEM. SPSS (SPSS, IL, USA) is used for statistical analysis. Statistical differences were considered significant when $p<0.05$. Survival curves were constructed according to the Kaplan-Meier method, and the survivals were compared by means of the log-rank test.

\section{Results}

\section{Molecular modeling and kinase inhibition profile of YL529}

The novel multi-kinase small-molecule inhibitor YL529 have been identified by the computer-aided drug design (CADD), chemical synthesis and high-throughput screening (HTS) methods in our lab, and in vitro kinase binding assay showed that YL529 inhibited VEGFR2 activity at $10 \mu \mathrm{M}$, and YL529 significantly inhibited VEGFR-3 activity by $97 \%$ at the same concentration [18]. In this study, we used computer simulation and computer-based molecular docking methods to further explore the interaction modes of YL529 with the kinase domain of VEGFR-3, and Fig. 1 
depicts a possible binding configuration of YL529 with VEGFR-3. Based on these studies, we can see that the $\mathrm{N}$-methylpicolinamide of YL529 forms strong hydrogenbond interactions with the CYS930 residue in the hinge region of VEGFR-3. The $\mathrm{N}$ atom in amide moiety of YL529 forms another important hydrogen-bond interaction with the GLU896 in the DFG region of VEGFR-3. Additionally, YL529 also forms pi-related interactions with ASP1055, LYS879 and VAL859, consistent with what we have previously observed in YL529-VEGFR-2 interaction [18].
YL529 inhibits proliferation, migration, invasion, and tube formation of HLECs in vitro

CCK-8 assay was used to evaluate the potential proliferation inhibition effect of HLECs after treated with YL529 or vehicle for $48 \mathrm{~h}$. Our data showed that YL529 inhibited the proliferation of HLECs, with IC50 value of $5.5 \mu \mathrm{M}$.

We next examined the effects of YL529 on HLECs migration using a wound healing migration assay [19]. The results showed that YL529 markedly decreased the number of migrating HLECs, as shown in Fig. 2a. At $2.5 \mu \mathrm{M}$, YL529 could inhibit the migration of cells by $44.3 \%$, and

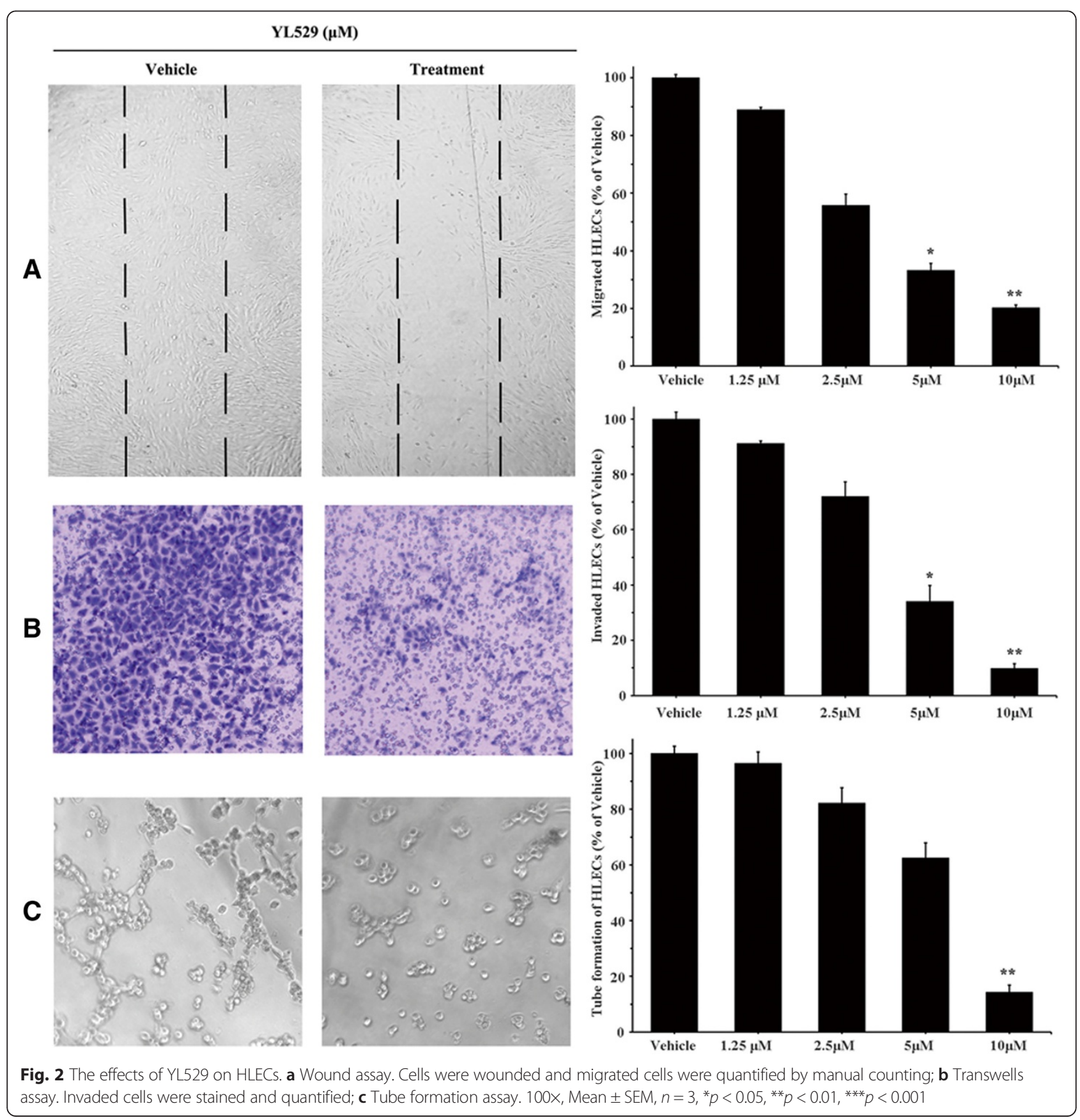


when the concentrations increased to $5 \mu \mathrm{M}$ or $10 \mu \mathrm{M}$, the inhibition of cells migration was increased to $66.8 \%$ or $79.8 \%$, respectively.

To further measure the effect of YL529 on HLECs invasion, we used a transwell assay and measured the number of HLECs that passed through a membrane barrier following treatment with various concentrations of YL529 [29]. As shown in Fig. 2b, compared with vehicle, $2.5 \mu \mathrm{M}$ YL529 could inhibit the invasion of cells by $28.0 \%$, and when concentrations of YL529 increased to $5 \mu \mathrm{M}$ or $10 \mu \mathrm{M}$, the inhibition of cell invasion was increased to $65.9 \%$ or $90.2 \%$, respectively.

To further understand the mechanism of the antilymphogenic effect of YL529, the antilymphogenic tube formation effect of YL529 was analyzed with cultured HLECs in vitro [30]. As shown in Fig. 2c, YL529 treatment at $10 \mu \mathrm{M}$ for $6 \mathrm{~h}$ strongly inhibited the formation of tube-like structures. Quantification showed that YL529 treatment inhibited tube formation by $85.7 \%$. In great contrast, HLECs without YL529 treatment spread and aligned with each other and formed a rich meshwork of branching capillary-like tubules within 6 h (Fig. 2c).

\section{YL529 inhibited the proliferation of VEGF-D-LL/2 cells}

VEGF-D-LL/2 cells were previously established by transfection with the pcDNA3.1 (+) expression vector containing mouse VEGF-D [31]. Similar to the previous data from our group, this study uses a VEGF-D antibody that detects the mature form of VEGF-D, because the fully mature cleaved form of $21 \mathrm{kD}$ has the greatest affinity for the receptors, and can bind and activate not only VEGFR-2 but also VEGFR-3. As shown in Fig. 3a, western blot analysis showed that the expression level of VEGF-D (21 kD) in VEGF-D-LL/2 cells was higher than the cells that were transfected with null-vectors (pcDNA-LL/2). This result confirmed that VEGF-D protein was constitutively up-regulated in VEGF-D-LL/2 cells. When VEGF$\mathrm{D}-\mathrm{LL} / 2$ cells and parental LL/2 cells were treated with YL529 or vehicle for $48 \mathrm{~h}$, the calculated corresponding IC50 value was $7.2 \mu \mathrm{M}$ and $9.6 \mu \mathrm{M}$, respectively. And we found that YL529 potently inhibited the proliferation of VEGF-D-LL/2 cells,

\section{Potential molecular mechanism of the effects of YL529 on HLECs and VEGF-D-LL/2 cells}

To gain in-depth insight into the molecular mechanism of anti-lymphangiogenesis effects of YL529, the expression levels of VEGFR-3/p-VEGFR-3 in HLECs were analyzed by western blotting in vitro. As shown in Fig. 3b, in HLECs, YL529 potently inhibited the phosphorylation of VEGFR-3 after $2 \mathrm{~h}$ of treatment, even though no change of the total expression of VEGFR-3 was observed. This data may provide a molecular mechanism of the previously observed specific effects of YL529 on HLECs.

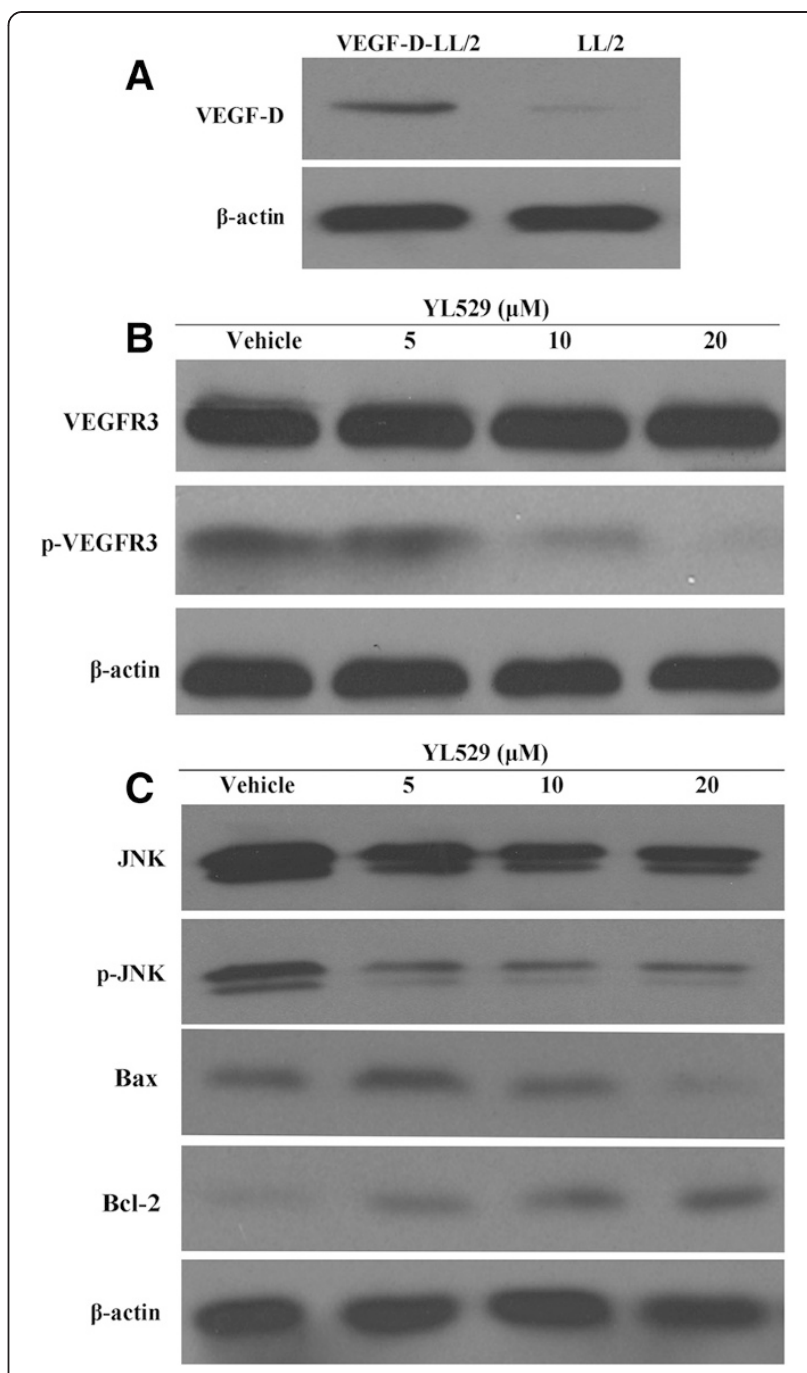

Fig. 3 Western blotting to probe the molecular mechanisms of effects of YL529 in vitro. a p-VEGFR3 in HLECs was detected after YL529 treatment by western blotting analysis. b The overexpression of VEGF-D in VEGF-D-LL/2 cells was confirmed by western blotting analysis. The molecular weight of full-length VEGF-D was 21 KD. c VEGF-D-LL/2 cell were treated with YL529, p-JNK/JNK, Bax, Bcl-2 were analyzed by western blotting analysis

Moreover, as shown in Fig. 3c, YL529 decreased the expression level of $\mathrm{p}-\mathrm{JNK}$ and Bax while increased the expression level of Bcl-2 in VEGF-D-LL/2 cells, which may explain the observed effects of YL529 on VEGF-D-LL/2 cells.

\section{Pharmacokinetics of YL529 in vivo}

To determine the pharmacokinetic characteristics of YL529 in mice, C57 BL/6 mice were treated with YL529 orally and the key pharmacokinetic parameters of YL529 were summarized in Table 1, i.e., after oral administration of YL529 at $150 \mathrm{mg} / \mathrm{kg}$, the peak plasma concentration (Cmax) was $18.03 \mu \mathrm{g} / \mathrm{ml}$, the time-to-peak concentration (Tmax) was $2 \mathrm{~h}$, the half-life $(\mathrm{t} 1 / 2 \mathrm{z})$ was $4.11 \mathrm{~h}$ and the $\mathrm{AUC}_{0 \rightarrow \infty}$ 
Table 1 Pharmacokinetic parameters of YL529 after oral administration with single dose of $150 \mathrm{mg} / \mathrm{kg}$ in mice

\begin{tabular}{lr}
\hline Pharmacokinetic parameter & Value \\
\hline $\mathrm{AUC}(0-\infty)\left(\mathrm{mg} / \mathrm{L}^{*} \mathrm{~h}\right)$ & 170.95 \\
$\mathrm{AUC}(0-\mathrm{t})\left(\mathrm{mg} / \mathrm{L}^{*} \mathrm{~h}\right)$ & 167.32 \\
$\mathrm{t} 1 / 2 \beta(\mathrm{h})$ & 6.03 \\
$\mathrm{~T} 1 / 2 \mathrm{a}(\mathrm{h})$ & 1.39 \\
$\mathrm{t} 1 / 2 \mathrm{z}(\mathrm{h})$ & 4.11 \\
$\mathrm{Cmax}(\mathrm{mg} / \mathrm{L})$ & 18.03 \\
$\operatorname{Tmax}(\mathrm{h})$ & 2.00
\end{tabular}

Data were expressed as the mean \pm SD compared with vehicle. SPSS (SPSS, IL) software was used for statistical analysis $\left(n=3 ;{ }^{*} p<0.05\right)$ was $170.95 \mathrm{mg} / \mathrm{L} \cdot \mathrm{h}$, which suggested that the oral absorption and bioavailability and the pharmacokinetics of YL529 in mice are highly desirable.

\section{YL529 inhibited tumor growth in VEGF-D-LL/2 tumors syngeneic models and extend the life span of affected mice}

Based on the safety profile and the in vivo pharmacokinetics of YL529, chronic oral administration of YL529 at daily dosages of $37.5 \sim 150 \mathrm{mg} / \mathrm{kg} /$ day was chosen to treat the mice with VEGF-D-LL/2 tumors (syngeneic s.c. and muscle models). We found that YL529 inhibited tumor growth in dose-dependent manner in both models.

Specifically, in s.c. model, as shown in Fig. 4a, there was a remarkable tumor volume reduction (66.22\%),

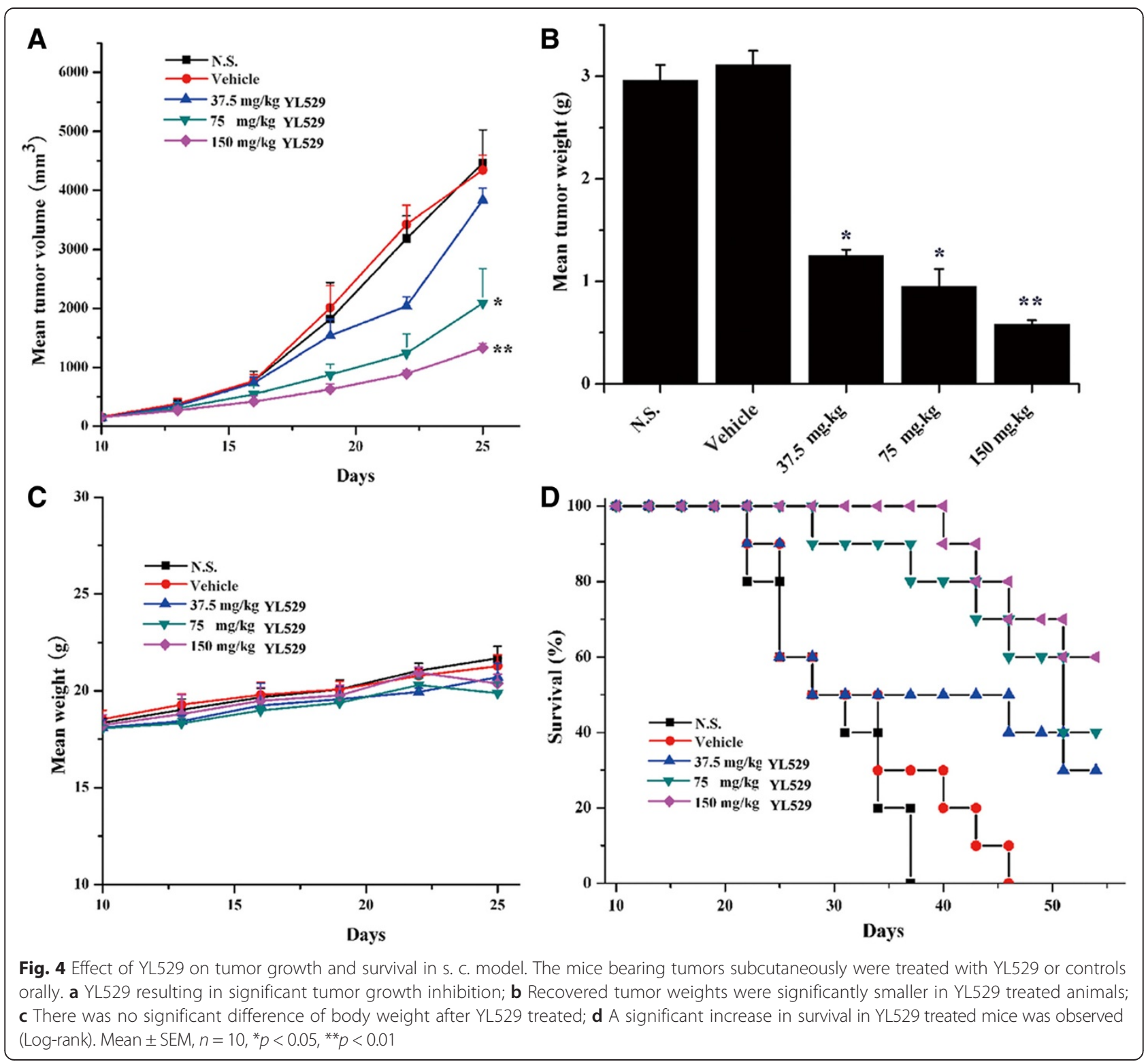


compared with the vehicle-treated group, and the final tumor weights showed similar pattern (Fig. 4b), while there was no loss of body weight at this dosage (Fig. 4c).
Furthermore, survival experiment in vivo showed that six out of ten animals in N.S. and vehicle groups died at 29 days (Mice were regarded as sacrificed when the

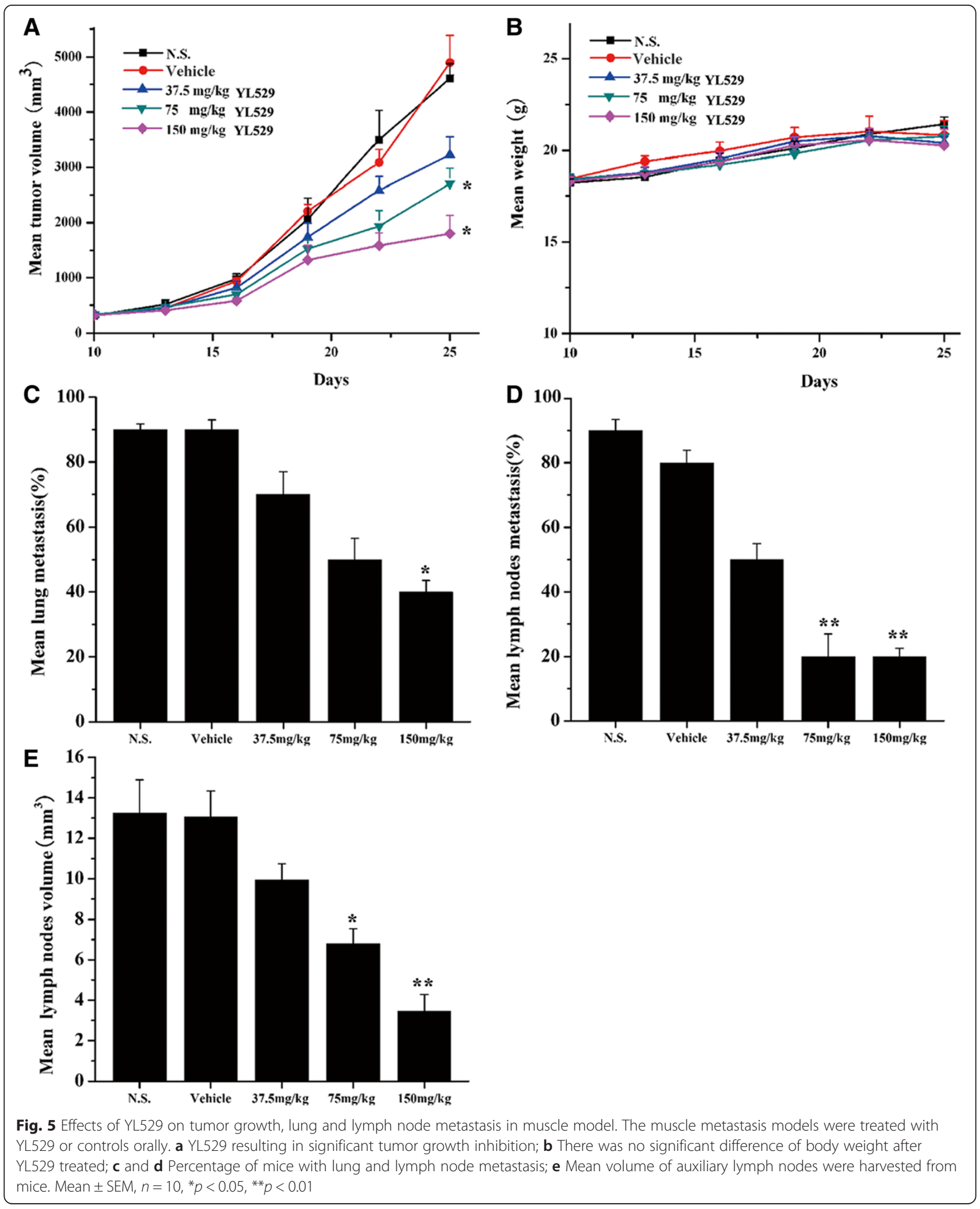


Table 2 The organs parameters of YL529 after oral administration $6000 \mathrm{mg} / \mathrm{kg}$ YL529 with a single dose in SD rats

\begin{tabular}{lllll}
\hline Index & $\begin{array}{l}\text { Vehicle } \\
\text { Female(\%) }\end{array}$ & $\begin{array}{l}\text { YL529 } \\
\text { Female (\%) }\end{array}$ & $\begin{array}{l}\text { Vehicle } \\
\text { Male (ơ) }\end{array}$ & $\begin{array}{l}\text { YL529 } \\
\text { Male (ơ) }\end{array}$ \\
\hline Heart (\%) & $0.41 \pm 0.08$ & $0.42 \pm 0.09$ & $0.43 \pm 0.05$ & $0.42 \pm 0.06$ \\
Liver (\%) & $3.11 \pm 0.10$ & $3.13 \pm 0.09$ & $3.09 \pm 0.10$ & $3.09 \pm 0.13$ \\
Spleen (\%) & $0.23 \pm 0.03$ & $0.25 \pm 0.03$ & $0.23 \pm 0.09$ & $0.24 \pm 0.04$ \\
Lung (\%) & $0.43 \pm 0.05$ & $0.43 \pm 0.06$ & $0.44 \pm 0.11$ & $0.42 \pm 0.09$ \\
Kidney (\%) & $0.37 \pm 0.06$ & $0.38 \pm 0.09$ & $0.36 \pm 0.08$ & $0.38 \pm 0.09$
\end{tabular}

Data were expressed as the mean \pm SD compared with vehicle. SPSS (SPSS, IL) software was used for statistical analysis $\left(n=10 ;{ }^{*} p<0.05\right)$

diameter of tumors reached about $20 \mathrm{~mm}$ ), while in YL529 treated groups, the life span of the mice have been significantly extended (Fig. $4 \mathrm{~d},{ }^{*} p<0.05$, by logrank test), i.e., $90 \%$ of mice survived to 40 days and $60 \%$ of mice in $150 \mathrm{mg} / \mathrm{kg}$ group survived to 54 days when we finished the survival observation.

Similar results were observed in the muscle model (Fig. 5a) (" $p<0.05)$. Moreover, it is worthy to stress that YL529 also significantly reduced the number of mice with lung metastasis and inguinal lymph node metastasis in the tumor muscle model, while there was no loss of body weight at this dosage at the end of drug administration (Fig. 5b). similarly, as shown in Fig. 5c, d and e $(" p<0.05, " p<0.01)$, the percent of mice with local lymph node metastasis in vehicle group could reach
$80 \% \sim 90 \%$, while reaching only $50 \%$ in $37.5 \mathrm{mg} / \mathrm{kg}$ group and $20 \%$ in $150 \mathrm{mg} / \mathrm{kg}$ group, respectively.

\section{Safety profile of YL529 in a preclinical study}

Currently, side effects are the prevalent shortcomings of anticancer drugs. In our current study, mice treated with YL529 at the daily dosage of $150 \mathrm{mg} / \mathrm{kg}$ for 18 days showed no obvious body weight loss or tissue damage, which is consistent with our previous reported [18]. Furthermore, we further evaluated the toxicity in SD rats treated by YL529 at the daily dosage of $6000 \mathrm{mg} / \mathrm{kg}$ for 14 days, and we found that there were no obvious weight changes of major organs (heart, liver, spleen, lung, kidney and brain) (Table 2), or obvious pathological damage, or other adverse effects (Data not shown).

\section{YL529 inhibited lymphangiogenesis in tumor tissues in additional to induce tumor cell apoptosis in vivo}

The immunohistochemical analysis and TUNEL apoptosis assays and LYVE-1 staining were performed to directly evaluate whether YL529 could inhibit the tumor lymphangiogenesis and induce tumor cell apoptosis in vivo. As shown in Fig. 6a, YL529 remarkably decreased the amount of lymphangiogenesis in tumor tissues at $150 \mathrm{mg} /$ $\mathrm{kg}$ for 14 days in VEGF-D-LL/2 tumor syngeneic model, as indicated by the LYVE-1 staining (34.71\% higher than the vehicle group in VEGF-D-LL/2 muscle model). Moreover, TUNEL assay found that there were a dramatic higher
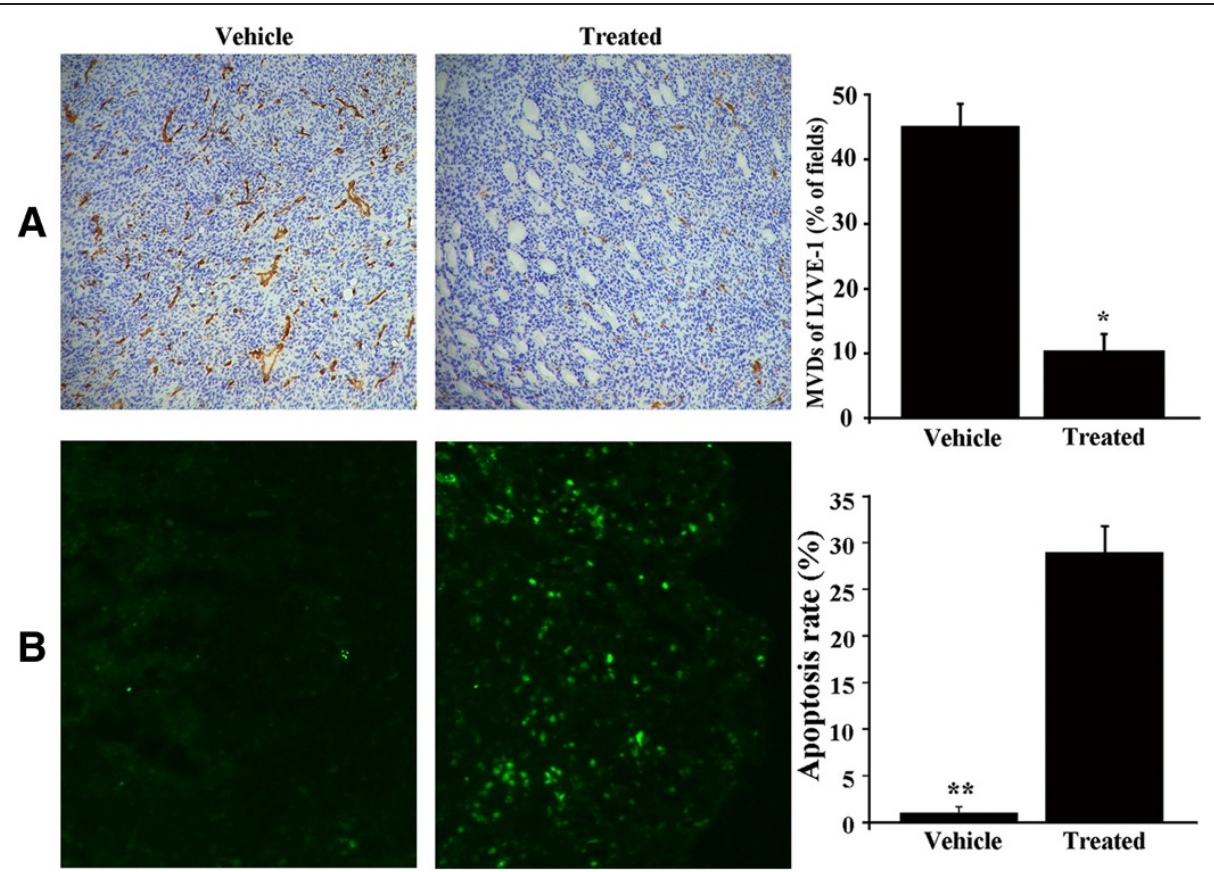

Fig. 6 Immunohistochemical analysis of LYVE-1 and TUNEL assay in vivo. Sections from vehicle group and $150 \mathrm{mg} / \mathrm{kg}$ YL529-treated tumor were collected. (a) LYVE-1 and (b) TUNEL were detected in the VEGF-D-LL/2 tumor model. Quantitative of the mean LYVE-1 and TUNEL-positive area counted at $\times 200$. Mean \pm SEM, 200x, $n=6,{ }^{*} p<0.05,{ }^{* *} p<0.01$ 
number of apoptotic tumor cells in the treated group (20-fold increase vs. vehicle), in s.c. model (Fig. 6b).

\section{Discussion}

Metastasis, particularly via the lymphatic system, is a common prognostic factor and critical process in the spread of solid cancer [2]. The VEGF/VEGFR pathway has been widely studied because VEGFR expression is strongly correlated with tumor metastasis progression and poor prognosis. Therefore, this pathway has been pursued as a therapeutic strategy for inhibition of lymphangiogenesis and metastatic in tumors,and some animal studies have shown that the expression of VEGF-D promotes lymphangiogenesis and metastatic spread of tumor cells via lymphatic in papillary thyroid carcinoma, lung cancer and gastric cancer [32-34]. However, most studies are based on cultured tumor cells. Little in vivo research has been done to correlate the high VEGF-D levels and lymph node metastasis in lung carcinoma, thus, it remains unclear whether expression of VEGF-D in culture accurately represents the in vivo situation.

Furthermore, since VEGF-D plays a remarkable role in up-regulating lymphangiogenesis and regional lymph node metastasis, other researchers in our group has established the mouse lymph node metastasis model by transfecting high expression VEGF-D into LL/2 Lewis lung carcinoma cells, and found that VEGF-D mainly bind to VEGFR-3 in lymphatic endothelial cells in vivo [31], and further study demonstrated that VEGFR-3 contributes to autocrine effects through VEGF-D-LL/2 cells [20].

YL529 was developed as a potential multikinase anticancer agent in our laboratory using CADD, HTS, de novo synthesis and has been reported to have a variety of pharmacological effects such as inhibiting tumor growth via antiangiogenesis and anti-proliferation in our previously study, especially it could block the activities of human umbilical vein endothelial cells (HUVEC) stimulated with $\mathrm{VEGF}_{165}$ previously [18]. And some researchers have reported that VEGFR-2 can also be expressed on lymphatic endothelial cells, while its expression levels were less than VEGFR-3 in lymphatic ECs, because VEGFR2 is the major effector of angiogenesis and regulates blood vessel growth of vascular ECs, not lymphatic ECs [35-37]. However, it is still possible that some of its inhibitory activities can be assigned to VEGFR-2, because YL529 is a multikinases inhibitor.

To further address some caveats, we determined that YL529 inhibits the proliferation and migration of highexpressing VEGF-D Lewis lung carcinoma LL/2 cells. Similar effects of YL529 were also observed in HLECs, which endogenously express high level of VEGFR-3. Consistent with this idea, we found that the anti-proliferation effect of YL529 was dependent on high VEGF-D expression, since YL529 has no as obvious effect on the parental LL/2 cells, which has low level of endogenous VEGF-D expression (Data not shown). Interestingly, high expression of VEGF-D has been found to correlate with the up-regulation of Fra-1, a member of the AP-1 family of transcription factors [38]. And AP-1 interacts with Fos and Jun protein family members, and the latter is regulated by ERK1/2 and JNKs, respectively.

Mechanistically, our result showed that YL529 downregulation of p-VEGFR-3 in HLECs cell in a dose-dependent manner, which is consistent with the computer-aided drug

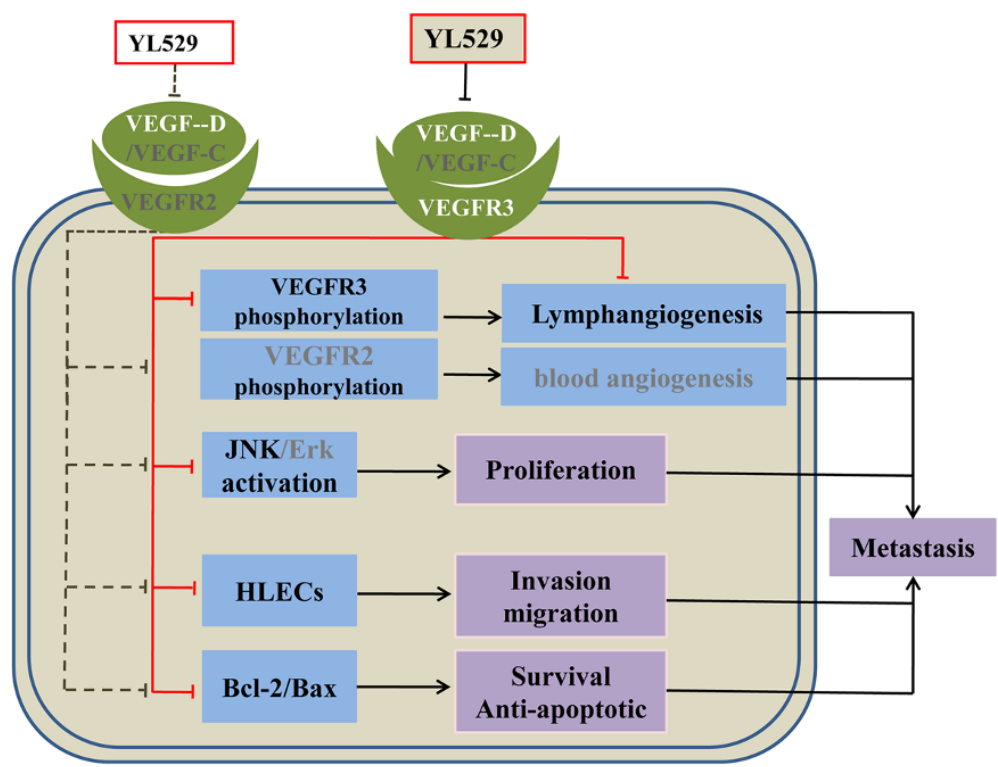

Fig. 7 The proposed underlying mechanisms of YL529 
design (CADD) and molecular reverse docking results. Furthermore, we found that YL529 also inhibited the phosphorylation of $\mathrm{JNK} 1 / 2$, while the total protein expression of JNK1/2 was not changed. Along this line, YL529 can also decrease the expression level of Bax and upregulate Bcl-2, which supported the idea of a potential pro-apoptotic effect of YL529. Taken together, it is reasonable to propose that YL529 could not only downregulated the levels of p-VEGFR-3, but also blocked the VEGFR-3 signaling pathway by interfering with the expression levels of $\mathrm{p}$-JNK and induced apoptosis via down-regulating Bax and up-regulating Bcl-2.

These results are further supported by the fact that YL529 inhibited the tumor-associated lymphangiogenesis and metastasis in mice harboring VEGF-D-LL/2 Lewis carcinoma in vivo, and by immunohistochemical staining showed that YL529-treated tumors displayed less LYVE-1 positive vessels compared with vehicle.

Our data has confirmed that YL529 exhibits the characteristics of blocking tumor metastasis at several different time points in the process of tumor lymphangiogenesis for the first time: (I) YL529 inhibits proliferation and induces apoptosis of in high expression VEGF-D tumor cells; (II) YL529 downregulates the expression of VEGFR-3 on HLECs and blockades the VEGFR-3 signaling pathways by interfering with the activation of JNK1/2; (III) YL529 interrupts and inhibits HLECs proliferation, invasion and tube formation; (IV) YL529 inhibits the tumor-associated lymphangiogenesis; (V) YL529 suppresses tumor cell migration and induces apoptosis. Moreover, there is a closely associated between lymph node metastasis and VEGFR-3 expression in several lewis lung carcinoma models. The binding of VEGF-D and VEGFR-3 could promote migration and proliferation of HLECs through JNKs signaling pathways. So blockade of the VEGFR-3 pathways could efficiently inhibit tumor lymphangiogenesis and metastasis. These results support the hypothesis that the therapeutic effect of YL529 on tumor lymphangiogenesis and metastasis could be attributed to direct inhibition of lymphangiogenesis in lung tumor models induced by VEGF-D and through down-regulation of VEGFR-3.

\section{Conclusions}

In conclusion, our results indicated that YL529 significantly inhibited the tumor-associated lymphangiogenesis and metastasis induced by VEGF-D in the established VEGF-D over-expressing Lewis lung carcinoma model (Fig. 7). Tumor growth was significantly retarded and prolonged life span was observed after YL529 treatment in tumor-bearing mice. We have demonstrated for the first time that YL529 suppresses tumor lymphangiogenesis and lymphatic metastasis by down-regulation of VEGF-D in VEGF-D-LL/2 cancer cells and inhibiting neogenesis and tube formation of lymphatic endothelial cells directly through the VEGFR-3 pathway. Our findings suggest that YL529 may be an attractive agent against lymphangiogenesis and metastasis. Moreover, YL529 has a novel chemical structure that is different from VEGFR-3 inhibitors in clinical use. In addition, YL529 was well tolerated by the host animal in therapeutically beneficial doses, making it a promised candidate for phase I clinical trials as an antilymphatic metastasis drug.

\section{Abbreviations}

HLECs: Human lymphatic endothelial cells; VEGF: Vascular endothelial growth factor; VEGF-C/D: Vascular endothelial growth factor-C/D; HUVECs: Human umbilical vein endothelial cells; HPLC: High performance liquid chromatography; NMR: Nuclear magnetic resonance; DMSO: Dimethyl-sulphoxide;

CMC-Na: Carboxymethylcellulose; ECM: Endothelial cell medium; FBS: Fetal bovine serum; ATCC: American type culture collection; OD: Optical density; PBS: Phosphate buffered saline; RIPA: Radioimmunoprecipitation assay; PVDF: Polyvinylidene fluoride; SDS-PAGE: Sodium dodecyl sulfate polyacrylamide gel electrophoresis; Cmax: The peak plasma concentration; Tmax: The time-to-peak concentration; t1/2z: The half-life; TUNEL: Terminal deoxynucleotidyl transferase mediated nick-end labeling.

\section{Competing interests}

The authors declare that they have no competing interests.

\section{Authors' contributions}

YZ X, WJ L, P Y, W P, CT W, YL Z, LT Y and SY Y were responsible for experimental design, interpretation of the results and writing the manuscript. WJ $L, Y Z X, M L L, Y L$ and $G B L$ performed the experimental procedures. $Y Z$ $X, W J L, P Y, N N M, S Y W$ and $H J L$ were responsible for the analysis of the data. LX K provided purely writing assistance. All authors read and approved the manuscript.

\section{Acknowledgements}

This work was supported by National Science \& Technology Major Project (2011ZX09102-001-013 and 2012ZX09501), National Natural Sciences Foundation of China (81272459 and 81402947), Natural Sciences Foundation of Anhui Province (1508085QH162), and the Grants for Scientific Research of BSKY from Anhui Medical University (XJ201315).

\section{Author details}

'Department of Pathophysiology, School of Basic Medicine, Anhui Medical University, 81\#, Mei Shan Road, Hefei 230032, China. ${ }^{2}$ State Key Laboratory of Biotherapy and Cancer Center, West China Hospital, West China Medical School, and Collaborative Innovation Center for Biotherapy, Sichuan University, 17\#, 3rd Section, Ren min South Road, Chengdu 610041, China. ${ }^{3}$ Department of Transfusion, the First Affiliated Hospital of Anhui Medical University, 81\#, Mei Shan Road, Hefei 230032, China. ${ }^{4}$ Department of Oncology, The People's Hospital of Guizhou Province, 83\#, Zhong Shan East Road, Guiyang 550004, China.

Received: 13 October 2014 Accepted: 19 May 2015

Published online: 18 July 2015

\section{References}

1. Hanahan D, Weinberg RA. Hallmarks of cancer: the next generation. Cell. 2011;144:646-74

2. Stacker SA, Williams SP, Karnezis T, Shayan R, Fox SB, Achen MG. Lymphangiogenesis and lymphatic vessel remodelling in cancer. Nat Rev Cancer. 2014;14:159-72.

3. Stacker SA, Caesar C, Baldwin ME, Thornton GE, Williams RA, Prevo R, et al. VEGF-D promotes the metastatic spread of tumor cells via the lymphatics. Nat Med. 2001;7:186-91.

4. Karaman S, Detmar M. Mechanisms of Iymphatic metastasis. J Clin Invest. 2014;124:922-8.

5. Cao Y. Emerging mechanisms of tumour lymphangiogenesis and lymphatic metastasis. Nat Rev Cancer. 2014;5:735-43.

6. Alitalo A, Detmar M. Interaction of tumor cells and lymphatic vessels in cancer progression. Oncogene. 2014;31:4499-508. 
7. Baldwin ME, Halford MM, Roufail S, Williams RA, Hibbs ML, Grail D, et al. Vascular endothelial growth factor $D$ is dispensable for development of the lymphatic system. Mol Cell Biol. 2005;25:2441-9.

8. Smith NR, Baker D, James NH, Ratcliffe $K$, Jenkins M, Ashton SE, et al. Vascular endothelial growth factor receptors VEGFR-2 and VEGFR-3 are localized primarily to the vasculature in human primary solid cancers. Clin Cancer Res. 2010;16:3548-61.

9. Achen $M G$, Jeltsch $M$, Kukk E, Mäkinen T, Vitali A, Wilks AF, et al. Vascular endothelial growth factor D (VEGF-D) is a ligand for the tyrosine kinases VEGF receptor 2 (FIk1) and VEGF receptor 3 (Flt4). Proc Natl Acad Sci U S A. 1998;95:548-53.

10. Karnezis T, Shayan R, Caesar C, Roufail S, Harris NC, Ardipradja K, et al. VEGF-D promotes tumor metastasis by regulating prostaglandins produced by the collecting lymphatic endothelium. Cancer Cell. 2012;14:181-95.

11. Zhang L, Zhou F, Han W, Shen B, Luo J, Shibuya M, et al. VEGFR-3 ligandbinding and kinase activity are required for lymphangiogenesis but not for angiogenesis. Cell Res. 2010;20:1319-31.

12. Skobe $M$, Hawighorst $T$, Jackson DG, Prevo R, Janes $L$, Velasco $P$, et al. Induction of tumor lymphangiogenesis by VEGF-C promotes breast cancer metastasis. Nat Med. 2007;7:192-8.

13. Han KY, Chang JH, Dugas-Ford J, Alexander JS, Azar DT. Involvement of lysosomal degradation in VEGF-C-induced down-regulation of VEGFR-3. FEBS Lett. 2014;588:4357-63.

14. Nwogu CE, Yendamuri S, Tan W, Kannisto E, Bogner P, Morrison C, et al. Lung cancer lymph node micrometastasis detection using real-time polymerase chain reaction. correlation with vascular endothelial growth factor expression. J Thorac Cardiov Sur. 2013;145:702-7.

15. Hecht JR, Trarbach T, Hainsworth JD, Major P, Jäger E, Wolff RA, et al. Randomized, placebo-controlled, phase III study of first-line oxaliplatin-based chemotherapy plus PTK787/ZK 222584, an oral vascular endothelial growth factor receptor inhibitor, in patients with metastatic colorectal adenocarcinoma J Clin Oncol. 2011;29:1997-2003.

16. Podar K, Anderson KC. Emerging therapies targeting tumor vasculature in multiple myeloma and other hematologic and solid malignancies. Curr Cancer Drug Targets. 2011;11:1005-24.

17. Wilhelm SM, Dumas J, Adnane L, Lynch M, Carter CA, Schütz G, et al Regorafenib (BAY 73-4506): a new oral multikinase inhibitor of angiogenic stromal and oncogenic receptor tyrosine kinases with potent preclinical antitumor activity. Int J Cancer. 2011;129:245-55.

18. Xu Y, Lin H, Meng N, Lu W, Li G, Han Y, et al. YL529, a novel, orally available multikinase inhibitor, potently inhibits angiogenesis and tumor growth in preclinical models. Brit J Pharmacol. 2013;169:1766-80.

19. Shibuya M. Vascular Endothelial Growth Factor (VEGF) and Its Receptor (VEGFR) Signaling in Angiogenesis: A Crucial Target for Anti- and Pro-Angiogenic Therapies. Genes Cancer. 2011;2:1097-105.

20. Jiang QQ, Fan LY, Yang GL, Guo WH, Hou WL, Chen L, et al. Improved therapeutic effectiveness by combining liposomal honokiol with cisplatin in lung cancer model. BMC cancer. 2008;8:242.

21. Hu J, Ye H, Fu A, Chen X, Wang Y, Chen X, et al. Deguelin-an inhibitor to tumor lymphangiogenesis and lymphatic metastasis by downregulation of vascular endothelial cell growth factor-D in lung tumor model. Int J Cancer. 2010;127:2455-66.

22. Hirakawa S, Brown LF, Kodama S, Paavonen K, Alitalo K, Detmar M. VEGF-Cinduced lymphangiogenesis in sentinel lymph nodes promotes tumor metastasis to distant sites. Blood. 2007:109:1010-7.

23. Xu YZ, Zheng RL, Zhou Y, Peng F, Lin HJ, Bu Q, et al. Small molecular anticancer agent SKLB703 induces apoptosis in human hepatocellular carcinoma cells via the mitochondrial apoptotic pathway in vitro and inhibits tumor growth in vivo. Cancer Lett. 2011;313:44-53.

24. Liang S, Fu A, Zhang Q, Tang M, Zhou J, Wei Y, et al. Honokiol inhibits HepG2 migration via down-regulation of IQGAP1 expression discovered by a quantitative pharmaceutical proteomic analysis. Proteomics. 2011;10:1474-83.

25. Goldberg L, Kloog Y. A Ras Inhibitor Tilts the Balance between Rac and Rho and Blocks Phosphatidylinositol 3-KinaseaDependent Glioblastoma Cell Migration. Cancer Res. 2006;66:11709-17.

26. Agarwal A, Covic L, Sevigny LM, Kaneider NC, Lazarides K, Azabdaftari G, et al. Targeting a metalloprotease-PAR1 signaling system with cell-penetrating pepducins inhibits angiogenesis, ascites, and progression of ovarian cancer. Mol Cancer Ther. 2008;7:2746-57.
27. Liu JY, Wei YQ, Yang L, Zhao X, Tian L, Hou JM, et al. Immunotherapy of tumors with vaccine based on quail homologous vascular endothelial growth factor receptor-2. Blood. 2003;102:1815-23.

28. Mikalsen LT, Dhakal HP, Bruland OS, Nesland JM, Olsen DR. Quantification of angiogenesis in breast cancer by automated vessel identification in CD34 immunohistochemical sections. Anticancer Res. 2011:31:4053-60.

29. Petrovic N, Schacke W, Gahagan JR, O'Conor CA, Winnicka B, Conway RE, et al. CD13/APN regulates endothelial invasion and filopodia fonnation. Blood. 2007;110:142-50.

30. Hertel J, Hirche C, Wissmann C, Ebert MP, Höcker M. Transcription of the vascular endothelial growth factor receptor-3 (VEGFR3) gene is regulated by the zinc finger proteins Sp1 and Sp3 and is under epigenetic control: transcription of vascular endothelial growth factor receptor 3. Cell Oncol (Dordr). 2014;37:131-45.

31. Wen J, Fu AF, Chen $\sqcup$, Xie XJ, Yang GL, Chen XC, et al. Liposomal honokio inhibits VEGF-D-induced lymphangiogenesis and metastasis in xenograft tumor model. Int J Cancer. 2009;124:2709-18.

32. Yasuoka H, Nakamura Y, Zuo H, Tang WH, Takamura Y, Miyauchi A, et al. VEGF-D expression and lymph vessels play an important role for lymph node metastasis in papillary thyroid carcinoma. Modern pathol. 2005;18:1127-33.

33. Matsumoto M, Roufail S, Inder R, Caesar C, Karnezis T, Shayan R, et al. Signaling for lymphangiogenesis via VEGFR-3 is required for the early events of metastasis. Clin Exp Metastasis. 2013;30:819-32.

34. Zhao YC, Ni XJ, Wang MH, Zha XM, Zhao Y, Wang S. Tumor-derived VEGF-C, but not VEGF-D, promotes sentinel lymph node lymphangiogenesis prior to metastasis in breast cancer patients. Med Oncol. 2012;29:2594-600.

35. Cleaver O, Melton DA. Endothelial signaling during development. Nat Med. 2003:9:661-8.

36. McCarty M, Wey J, Stoeltzing O, Liu W, Fan F, Bucana C, et al. ZD6474, a vascular endothelial growth factor receptor tyrosine kinase inhibitor with additional activity against epidermal growth factor receptor tyrosine kinase, inhibits orthotopic growth and angiogenesis of gastric cancer. Mol Cancer Ther. 2004;3:1041-8.

37. Gaengel K, Genove G, Armulik A, Betsholtz C. Endothelial-mural cell signaling in vascular development and angiogenesis. Arterioscl Throm Vas Biol. 2009;29:630-8.

38. Debinski W, Slagle-Webb B, Achen MG, Stacker SA, Tulchinsky E, Gillespie GY, et al. VEGF-D is an X-linked/AP-1 regulated putative onco-angiogen in human glioblastoma multiforme. Mol Med. 2001;7:598-608.

\section{Submit your next manuscript to BioMed Central and take full advantage of:}

- Convenient online submission

- Thorough peer review

- No space constraints or color figure charges

- Immediate publication on acceptance

- Inclusion in PubMed, CAS, Scopus and Google Scholar

- Research which is freely available for redistribution 